\title{
DER ELEFANT IM RAUM. ETHNOGRAPHISCHES FORSCHUNGSDATENMANAGEMENT ALS HERAUSFORDERUNG FÜR REPOSITORIEN
}

\section{von Igor Eberhard \& Wolfgang Kraus}

Zusammenfassung: Von den meisten Fördergebern im wissenschaftlichen Bereich wird heute Forschungsdatenmanagement erwartet. Eine Grundlage dessen ist die Zugänglichkeit und Nachhaltigkeit der Daten. FAIR Data Principles und möglichst auch Open Access zu Daten gelten dabei als wesentliche Kriterien. Im Zusammenhang mit ethnographischen Forschungsdaten stößt man jedoch, wie durch das an der Universität Wien angesiedelte Pilotprojekt „Ethnographische Datenarchivierung“ aufgezeigt, bald aufgroße Herausforderungen für Repositorien, die den Möglichkeiten für Verfügbarmachung und Nachnutzung von Daten Grenzen setzen. Neben fachspezifischen und praktischen Gründen sind es vor allem rechtliche und ethische Anforderungen, die neue Wege erfordern. Der Artikel geht ethnographischer Forschung im Spannungsfeld von fachspezifischen, rechtlichen und ethischen Anforderungen für Repositorien nach.

Schlüsselwörter: Ethnographische Datenarchivierung; Ethnographie; Forschungsdatenmanagement; Repositorien; Forschungsethik; Nachhaltigkeit; Kultur- und Sozialanthropologie

\section{THE ELEPHANT IN THE ROOM: CHALLENGES FOR ETHNOGRAPHIC DATA MANAGEMENT REPOSITORIES}

\begin{abstract}
Today's scientific research funding is often inextricably linked to data management which in turn presupposes data accessibility and sustainability. Common criteria are FAIR Data Principles and open access dissemination of research data. Yet, the University of Vienna-based pilot project 'Ethnographic data archiving' highlights the limitations which arise in the process of establishing ethnographic data management repositories. These do not only result from practical and subject-specific challenges but also concern current legal and ethical requirements to organizing repositories. The article aims to discuss some of the challenges and limitations of creating and sustaining repositories for ethnographic fieldwork data.
\end{abstract}

Keywords: ethnographic data archiving; ethnography; research data management; repositories; research ethics; social and cultural anthropology

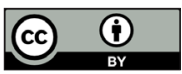


DOI: https://doi.org/10.31263/voebm.v71i1.2018

\section{Inhalt}

\section{Einleitung}

2. Der Elefant im Raum

3. Charakteristika ethnographischer Forschung

4. Herausforderungen für das Forschungsdatenmanagement

5. Ethische Anforderungen an das ethnographische Forschen

6. Das Projekt Ethnographische Datenarchivierung

7. Für eine Zukunft ohne Elefant im Raum

\section{Einleitung}

Die meisten wissenschaftlichen Paradigmata zeigen nach einiger Zeit ihre blinden Flecke - oder zumindest ihre Schwächen. Spätestens seit der Postmoderne mit ihren scientific turns ist das deutlich geworden. Einige der neuen Paradigmata haben ihre längerfristige Berechtigung unter Beweis gestellt und neue Perspektiven und Einsichten ermöglicht. Weitgehend konstant geblieben sind zugleich Ansprüche wie die Transparenz und Nachhaltigkeit im Umgang mit Quellen und Daten. Seit einigen Jahren gibt es verstärkt paradigmatische Ansätze, die darauf abzielen, diese Ansprüche zu vertiefen und möglichst auch eine zugänglichere Wissenschaft zu ermöglichen. Dazu zählen Forschungsdatenmanagement, Open Access und die FAIR Data-Prinzipien, also die Forderung nach Daten, die auffindbar, zugänglich, interoperabel und wiederverwendbar sind (Force11 2017).

Basis jedweder wissenschaftlichen Arbeit ist die Quellentransparenz und deren Nachvollziehbarkeit. Dafür sollen Forschungsdaten zugänglich sein. In der Praxis war das bisher nicht immer ausreichend der Fall. Immer noch sind etwa Quellen in abgelegenen, kleinen Archiven oder kaum zugänglichen Sammlungen aufbewahrt oder in Büchern mit geringer Auflage publiziert; Forschungsdaten waren und sind oft schlecht gesichert direkt bei den Forschenden gespeichert (vgl. Imeri 2017: 168). Institutionen wie Bibliotheken, Archive, Museen oder gelegentlich auch private Sammlungen konnten hier bis zu einem gewissen Grad Abhilfe schaffen. Man musste jedoch Mühe, Geduld und Insiderwissen aufwenden, um das gesuchte Material aufzufinden und Zugang dazu zu erhalten. So blieb der Zugang zu Quellen und Daten ein Stück weit esoterisch und elitär.

Ein durchdachtes Forschungsdatenmanagement, Open Access und FAIR Data bieten daher gute Chancen, Wissenschaft effizienter und leich- 
ter zugänglich zu machen. Die großen Fördergeber wie der Fonds zur Förderung der wissenschaftlichen Forschung (FWF) in Österreich oder die Deutsche Forschungsgemeinschaft (DFG) und viele andere verlangen aus diesem Grund Forschungsdesigns, die den genannten Kriterien gerecht werden (vgl. etwa RatSWD 2018a). Doch bei der praktischen Umsetzung zeigt sich in manchen Bereichen, dass diese Forderungen noch nicht ausreichend konkret oder durchdacht sind oder an der praktischen Forschungsrealität vorübergehen; anders gesagt, dass ihre Anpassung an diese Realität erst geleistet werden muss. Im Bereich ethnographischer Forschung ist das mit besonderer Deutlichkeit zu sehen.

Zugleich zeigt sich, dass derartige Forderungen nicht allein auf die Anpassung des Umgangs mit Daten an die digitale Forschungspraxis der Gegenwart abzielen, die in programmatischen Erklärungen (vgl. etwa TIB 2016; Force11 2017) meist im Vordergrund steht. Es ist unschwer zu erkennen, dass zugleich eine Neubestimmung der Rolle von Wissenschaft in der Gesellschaft stattfindet. Hinter den expliziten gesellschaftlichen Diskursen zur Effizienz und Verwertbarkeit der akademischen Wissensproduktion stehen aber, weniger offenkundig, auch implizite Annahmen, die es offenzulegen und zu überprüfen gilt - so etwa im Zusammenhang mit den anvisierten Zeithorizonten einer Nachnutzung von Forschungsdaten, die bei Strategien zur Langzeitarchivierung von Daten von kritischer Bedeutung sind.

\section{Der Elefant im Raum}

In gewissen Forschungsbereichen besteht also eine deutliche Diskrepanz zwischen den Anforderungen und Wünschen der Fördergeber und anderer Institutionen, aber auch den Möglichkeiten, die Repositorien für ein Forschungsdatenmanagement bieten, auf der einen Seite und den fachspezifischen Forschungspraktiken und -notwendigkeiten auf der anderen. Die Metapher vom „Elefant im Raum“ eignet sich gut, um diese Problematik zu beschreiben: Es ist offensichtlich, dass es in den Anforderungen ans Datenmanagement Widerspüchlichkeiten (wie etwa Open Data vs. Datenschutz), Lücken, ungelöste Probleme und ethische Unvereinbarkeiten gibt, aber sie werden vorerst ausgeklammert oder nur zögernd und nach und nach bedacht. Der Elefant im Raum bleibt vorhanden - und versperrt möglicherweise den Blick auf Lösungen.

Forschungsdatenmanagement funktioniert in der Praxis in manchen Disziplinen und mit manchen Methoden besser und unproblematischer als 
in anderen. In den Sozialwissenschaften sind insbesondere bei qualitativen Daten Lösungen allenfalls in Ansätzen erkennbar. Die Ankündigung des Workshops „Archivierung und Zugang zu qualitativen Daten“ des deutschen Rates für Sozial- und Wirtschaftsdaten im April 2018 verdeutlicht diese Ratlosigkeit: „Die institutionelle Struktur für die Archivierung und Verfügbarmachung qualitativer Forschungsdaten ist in Deutschland immer noch ein Desiderat. Speziell für qualitatives Material bestehen bislang wenige Möglichkeiten, dieses nach Projektabschluss langfristig und nach haltig zu archivieren" (RatSWD 2018b).

In Österreich gab es durch das sozialwissenschaftliche Datenarchiv WISDOM bereits ab 2007 erste durchdachte Überlegungen für eine Langzeitarchivierung qualitativer Daten (vgl. Smioski 2011; 2012), die jedoch nach der Einstellung des Archivs nicht weitergeführt wurden. Seine Datensätze sind in das 2017 etablierte Austrian Social Science Data Archive (AUSSDA o.J., https://aussda.at/) übergegangen, dessen Schwerpunkt aber klar auf quantitativen Daten liegt (AUSSDA o.J.). Insbesondere für Daten aus ethnographischen Erhebungen mit ihren spezifischen Herausforderungen sind Lösungen im gesamten deutschsprachigen Raum noch in weiter Ferne.

\section{Charakteristika ethnographischer Forschung}

Der methodische Ansatz der ethnographischen Feldforschung wurde in jenem Fach entwickelt, das an der Universität Wien die am internationalen Gebrauch orientierte Bezeichnung Kultur- und Sozialanthropologie trägt, während im deutschen Sprachraum generell noch die Bezeichnung Ethnologie vorherrscht. Ethnographische Ansätze sind mittlerweile - in angepasster Form - auch von anderen sozial- und kulturwissenschaftlichen Fächern aufgegriffen worden.

Der ethnographische Zugang beruht auf der Erhebung vor allem qualitativer Daten im engen und meist langfristigen persönlichen Kontakt zwischen Forschenden und Beforschten. In der Alltagswelt der Beforschten werden Gespräche und Interviews, häufig in den lokalen Sprachen, geführt. Darüber hinaus werden Beobachtungsnotizen und -protokolle sowie Feldtagebücher geschrieben, Skizzen angefertigt und häufig auch Fotos oder Tonaufnahmen gemacht. Wichtige Charakteristika ethnographischer Forschung sind ein flexibler Methodenpluralismus sowie die grundlegende Offenheit, die methodischen Zugänge und Vorannahmen, mit denen die Forschenden ins Feld eintreten, an die dort vorgefundenen Verhältnisse anzupassen. Daraus, aber auch aus der Abhängigkeit der Forscherln von 
der aktiven Mitwirkung der Forschungssubjekte, ergeben sich Herausforderungen im Hinblick auf die Planbarkeit der Forschung im Allgemeinen und den Umgang mit Forschungsdaten im Besonderen.

Am deutlichsten zeigen sich die spezifischen Herausforderungen für Datenmanagement-Strategien, die dem ethnographischen Zugang und seinen Eigenarten angepasst sind (vgl. dazu Imeri 2017), in den klassischen Forschungsfeldern der Kultur- und Sozialanthropologie. Im Mittelpunkt anthropologischer Wissensproduktion stand lange die Forschung in zum Teil schwer zugänglichen, räumlich weit entfernten Communities außerhalb der industrialisierten Welt. Oft fanden die Forschungen in einem koIonialen Kontext statt, in dem die Forschenden, ob willentlich oder nicht, selbst zu einem Teil des kolonialen Herrschaftsapparats und seiner Unterdrückungsmechanismen wurden.

Auch wenn sich das Profil und die Fragestellungen des Faches in den letzten Jahrzehnten gewandelt haben, führen AnthropologInnen weiterhin lang andauernde Feldforschungen bei indigenen, zumeist kleinzähligen Gruppen durch - oder sie übertragen das Prinzip der intensiven dialogischen Forschung zu alltäglichen Lebenszusammenhängen der Forschungssubjekte auf Fragestellungen zur eigenen oder anderen europäischen Gesellschaften. Daraus ergeben sich spezifische Anforderungen an den Umgang mit ethnographischem Datenmaterial, in denen ethische und rechtliche Fragen eine zentrale Rolle spielen.

Die auf dem Vertrauensverhältnis zwischen Forschenden und Beforschten aufbauende Arbeit in oft sehr überschaubaren sozialen Feldern und die Arbeit mit kritischen personenbezogenen Daten (etwa zu politischen oder religiösen Ansichten, Krankheiten, Korruption, Genozid etc.) verlangt besondere Sensibilität im Umgang mit den Daten. Da die Forschenden oft längere Zeit in intensiver Interaktion im Feld zubringen, ist es außerdem notwendig, das Verhältnis von Nähe und Distanz zu den Beforschten zu reflektieren - was sich auch in Teilen der Daten wie Feldtagebüchern niederschlägt. Feldforschung ist und war immer auch eine persönliche Grenzerfahrung (vgl. etwa Caton 1990). In einer unbekannten Umgebung mit zunächst unbekannten Menschen eine mehr- oder vielmonatige Feldforschung durchzuführen ist nicht einfach. Sie schafft sehr viel Nähe, jedoch manchmal auch Befremden. Neben der eigentlichen Datenerhebung und der Reflexion der eigenen Positionierung erfordert sie auch eine gewisse Distanz. Zusätzlich zu der Arbeit an den Daten und mit den Beforschten ist daher auch die konsequente Arbeit an sich selbst und die Analyse des eigenen Zugangs erforderlich (vgl. Breidenstein et al. 2015). Ethnographische Forschung benötigt also weit mehr als nur Datenmaterial. Wie aber kann 
der notwendige Prozess der Selbstreflexion im Datenmanagement abgebildet werden?

\section{Herausforderungen für das Forschungsdatenmanagement}

Aus alledem ergibt sich, dass das Verfügbarmachen von ethnographischen Daten besondere Herausforderungen mit sich bringt. Ein uneingeschränkter Zugang zu Forschungsdaten aus einem sozialen Feld, in dem alle Akteure einander kennen und damit identifizierbar sind, führt das Rechtsprinzip des Schutzes personenbezogener Daten ad absurdum und kann in Abhängigkeit von den jeweiligen Forschungsfragen - zur Stigmatisierung und persönlichen Gefährdung der Forschungssubjekte führen; er steht damit auch mit den grundlegendsten Anforderungen der Forschungsethik im Widerspruch. Da ethnographische Daten nur aus dem sozialen und kulturellen Kontext - also ergänzenden Informationen zu den Rahmenbedingungen - heraus interpretierbar sind, ist in vielen Fällen auch eine Pseudonymisierung ohne Verlust an Datendichte schwierig, eine Anonymisierung gänzlich unmöglich. Im Zusammenhang mit Themen wie politischem Aktivismus und Widerstand, Devianz, sexueller Orientierung können die Beforschten zudem nicht nur einer Gefährdung durch ihr unmittelbares soziales Umfeld, sondern auch durch staatliche Institutionen und Systeme ausgesetzt sein.

Bei solchen Daten stellt sich daher die Frage ob und unter welchen Voraussetzungen sie archiviert und verfügbar gemacht werden können. Ein gänzlich offener Zugang wird nur in manchen unproblematischen Fällen vertretbar sein. Die Grundsätze der Auffindbarkeit, Zugänglichkeit und Nachnutzbarkeit, wie sie durch die FAIR Data-Prinzipien gefordert werden, lassen sich somit nur bedingt umsetzen. Selbst wenn der Zugang zu den Daten beschränkt ist, können bereits die Metadaten, sollen sie ihren eigentlichen Zweck erfüllen, kritische Informationen preisgeben. Werden andererseits die Metadaten so allgemein gehalten, dass sie nichts Personenbezogenes verraten, dann riskiert man einen Verlust an Datendichte bis hin zur Unbrauchbarkeit.

Unabhängig von der Frage des Umgangs mit Metadaten braucht es für ethnographische Forschungsdaten jedenfalls gestaffelte Zugangsregelungen, die von uneingeschränkter Öffentlichkeit bis zu ausschließlicher Safe Room-Nutzung oder langfristigen Sperren reichen können (für ein ausgefeiltes Modell dieser Art s. Sterzer et al. 2018). Die Repositorien sind freilich auf solche Anforderungen meist noch nicht vorbereitet. 


\section{Ethische Anforderungen an das ethnographische Forschen}

Weitere Herausforderungen ergeben sich aus den notwendigen ethischen Standards anthropologischer Forschung. Diese erfordern eine Reflexion der Machtstrukturen, in welche Forschung unweigerlich eingebunden ist, und verbieten es den Forschenden, den Beforschten und ihren Interessen durch ihre Forschungspraxis, ihre Daten oder ihre Forschungsergebnisse Schaden zuzufügen (vgl. auch DGSKA 2008). Eine weitere ethische Anforderung an die ethnographische Arbeit betrifft den respektvollen Umgang auf Augenhöhe mit den Beforschten in den jeweiligen source communities, ohne deren Mitwirkung und Mitgestaltung es keine ethnographische Forschung geben kann.

Das impliziert auch die Weitergabe - oder eher Rückgabe - der Daten, Publikationen etc. an die Beforschten (Stichwort Reziprozität). Diese selbst werden immer öfter zu kritischen Leserlnnen und KommentatorInnen der sie betreffenden Forschungen. Folglich sollten sie nicht nur in die Datenerhebung, sondern auch in das Forschungsdatenmanagement einbezogen werden.

In einigen Fällen gehen die Forderungen der Beforschten sogar noch weiter. Sie nehmen direkten Einfluss auf die Forschungsfrage, das Forschungsdesign und den Forschungsprozess, sowie auf die Analyse, Archivierung und Zugänglichkeit der entstandenen Daten und Ergebnisse. VertreterInnen der communities entscheiden, zum Teil zusammen mit indigenen WissenschaftlerInnen, Universitäten oder Institutionen vor Ort, wer was auf welche Weise beforschen kann - oder eben nicht. Das betrifft beispielsweise in verschiedenen Ausprägungen Forschungen in Neuseeland, Australien, USA und Kanada. Besonders in Kanada sind die Standards für den Umgang mit der indigenen Bevölkerung sehr hoch. Die für die Umsetzung dieser Standards verantwortliche Institution, das Panel on Research Ethics, legt die Voraussetzungen für Forschungen mit Menschen und insbesondere mit indigenen Gruppen genau fest: "[This chapter] accords respect to Aboriginal peoples' knowledge systems by ensuring that the various and distinct world views of Indian, Inuit and Métis peoples are represented in planning and decision making, from the earliest stages of conception and design of projects through to the analysis and dissemination of results. It affirms respect for community customs and codes of research practice to better ensure balance in the relationship between researchers and participants, and mutual benefit in researcher-community relations. [...] The desire to conserve, reclaim and develop knowledge specific to First Nations, Inuit and Métis communities, and to benefit from contemporary applications of 
traditional knowledge, is a motivating force in community initiatives to assume a decisive role in research. The guidance provided in this chapter is based on the premise that engagement with community is an integral part of ethical research involving Aboriginal peoples" (PRE 2018).

Wenn eine Forschungsinfrastrukur bzw. Repositorien genutzt werden sollen, müssen diese die Einhaltung dieser Grundsätze gewährleisten. Konkrete Forderungen können zum Beispiel die Mitsprache bei der Erstellung eines Forschungsdatenmanagementplans, die Wahl des Repositoriums, die Administration desselben oder auch das potentielle Löschen der Daten betreffen. Verstöße gegen diese ethischen Ansprüche oder die daraus resultierenden Regeln und gesetzlichen Vorgaben diskreditieren ethnographisch Forschende.

Erschwert wird der Umgang mit diesen ethischen Anforderungen jedoch durch deutlich variierende Vorgaben in verschiedenen Ländern. Eine weitere Schwierigkeit ergibt sich aus der Frage, wer auf Grundlage welcher Legitimation als Vertreterln der beforschten Gruppe agieren kann: Wer gegenüber den Forschenden als Vermittlerln bzw. Sprecherln auftritt, das kann durchaus kontrovers gesehen werden. Verschärft werden kann diese Problematik fallweise noch durch die Intervention (mehr oder weniger) externer Akteure wie etwa religiöser oder staatlicher Institutionen.

Auch das Fehlen von vertrauenswürdigen oder dauerhaft vorhandenen Institutionen oder RepräsentantInnen (etwa in Kriegs- oder Krisengebieten) kann, ebenso wie die Abhängigkeit solcher Institutionen oder RepräsentantInnen von Kolonial- oder Besatzungsmächten (wie in Tibet, Irian Jaya oder auch ein Stück weit in Hawaii) kann zum Problem für die Forschungsarbeit werden. In solchen Fällen wird die Frage nach einer nachhaltigen Lösung des Zugangs zu Daten besonders problematisch. Es kann auch kontraproduktiv sein, Daten überhaupt sichtbar und damit nutzbar zu machen. In Krisengebieten wie in Afghanistan oder im Irak kann allein die Lokalisierung von Kulturgütern zu ihrer Zerstörung, die Identifizierbarkeit von InformantInnen zu ihrem Tod führen.

Ethnographische Forschung trägt also dazu bei, die Grenzen von Forschungsdatenmanagement, Open Access und FAIR Data aufzuzeigen. Für die meisten der angerissenen Problemfelder gibt es noch keine praktikablen Lösungen. Das Pilotprojekt „Ethnographische Datenarchivierung“ der DLE Bibliotheks- und Archivwesen am Institut für Kultur- und Sozialanthropologie der Universität Wien (EDA o.J., http://eda.univie.ac.at/) setzt sich seit 2017 mit diesen und anderen Herausforderungen auseinander. 


\section{Das Projekt Ethnographische Datenarchivierung}

In diesem für den deutschsprachigen Raum neuartigen Pilotprojekt versuchen die MitarbeiterInnen im engen Austausch mit anderen Forschenden und insbesondere mit den Datengeberlnnen, Lösungen, Strategien und Best Practice-Modelle zur nachhaltigen Archivierung und Nachnutzung von ethnographischen Forschungsdaten zu erarbeiten. Das Projekt ist Anfang 2017 eingerichtet worden und ist in der ersten Projektphase für zwei Jahre angesetzt. Sein Ziel ist es, in Abstimmung mit analogen universitären, nationalen und internationalen Initiativen Grundlagen für eine Datenmanagement-Strategie zu erarbeiten, die den Bedürfnissen und Besonderheiten ethnographischer Forschung angepasst ist. Die dabei gemachten Erfahrungen sollen in der Folge auch anderen Fächern zu Gute kommen, die mit ähnlichen Problemen kämpfen. In der ersten Phase stehen Datenbestände im Vordergrund, die von langjährigen Forschenden am Institut für Kultur- und Sozialanthropologie (IKSA) im Verlauf ihrer akademischen Karrieren erhoben worden sind. Mittelfristig soll diese derzeit eher historische Orientierung durch ein forschungsbegleitendes Datenmanagement ergänzt werden.

Längerfristiges Endziel ist das Einbringen der historischen, gegenwärtigen und zukünftigen ethnographischen Datenbestände der am IKSA Forschenden und anderer interessierter FachkollegInnen in ein digitales Repositorium mit internationaler Sichtbarkeit und Zugänglichkeit. Aufgrund der Anbindung des Projektes an das Bibliotheks- und Archivwesen wurde das ebenso dort angesiedelte Repositorium zur dauerhaften Sicherung von digitalen Beständen an der Universität Wien, PHAIDRA, dafür ausgewählt (Phaidra o.J., https://phaidra.univie.ac.at/).

Die unmittelbaren Projektergebnisse sollen eine fundierte Abschätzung der bestehenden Archivierungsbedürfnisse, Datenarten und -mengen sowie des erforderlichen Arbeits- und Mittelaufwandes für eine langfristige und nachhaltige Archivierungsstrategie ermöglichen und so eine wesentliche Grundlage für ein umfassendes Forschungsdatenmanagement in der Kultur- und Sozialanthropologie und anderen sozial- und kulturwissenschaftlichen Fächern liefern.

Ein erstes Ergebnis des Projekts ist die intensive Zusammenarbeit mit dem Fachinformationsdienst Sozial- und Kulturanthropologie an der Humboldt-Universität zu Berlin, wo mit Nachdruck an disziplin-spezifischen Fragen wie den oben gestellten gearbeitet wird (Imeri 2017; Sterzer et al. 2018), sowie mit verschiedenen Wiener Institutionen. Hier gibt es vor allem mit dem Institut für Europäische Ethnologie, dem Center for 
Interdisciplinary Research and Documentation of Inner and South Asian Cultural History und dem Projekt „Sammlungsideologie und Geschichtsschreibung" am Institut für Theater-, Film- und Medienwissenschaft (alle an der Universität Wien), dem Phonogrammarchiv und dem Institut für Sozialanthropologie der Österreichischen Akademie der Wissenschaften, dem PHAIDRA-Team sowie der Sammlungsbeauftragten der Universität Wien einen regelmäßigen Austausch.

Durch den Einsatz von PHAIDRA werden gleichzeitig auch die Möglichkeiten und Beschränkungen des Repositoriums ausgelotet, wo es bisher noch wenig nachhaltige Erfahrungen im Umgang mit ethnographischen und qualitativen Daten gibt. Daraus resultiert als zweites Zwischenergebnis, dass die spezifischen, durch Ethik und Reziprozität begründeten Anforderungen einer ethnographischen Datenarchivierung PHAIDRA auf dem derzeitigen Stand an seine Grenzen bringen. Durch die intensive Zusammenarbeit mit dem PHAIDRA-Team kann das Projekt EDA jedoch auf die Weiterentwicklung dieses Digital Asset Management Systems direkten Einfluss nehmen. Somit ergibt sich aus dieser Zusammenarbeit - eine entsprechende personelle und finanzielle Ausstattung vorausgesetzt - die Chance, hier gemeinsam Standards zu setzen.

Drittes vorläufiges Ergebnis ist: Die bislang fehlende Strategie der Universität Wien für einen nachhaltigen und ethisch fundierten Umgang insbesondere mit qualitativen Forschungsdaten erschwert die Etablierung von Lösungen. Solche Lösungen können aber nicht nur von einzelnen Repositorien oder Institutionen kommen, sondern brauchen einen breiten Austausch. Ohne die Zusammenarbeit mit anderen Institutionen, Forschungsproponenten, Fördergebern etc. werden nur weitere metaphorische Elefanten geschaffen. Wirkliche Lösungen wird nur eine gemeinsam erarbeitete Strategie bieten, die sich jedoch nicht über die spezifischen Anforderungen einzelner Disziplinen hinwegsetzen darf. Hier sind die Fachverbände aufgerufen, Stellung zu beziehen, und das jetzt - also zu einem Zeitpunkt, da die großen Akteure noch zuhören, die Spielregeln noch in Aushandlung sind.

Um diesen Austausch auf der Graswurzelebene voranzutreiben, wurde in der Folge des ersten EDA-Workshops zum Thema „Gibt es ein Weiterleben der Daten nach der Forschung? Ethnographische Forschungsdaten, Forschungsdatenmanagement und Sammlungsstrategien als Herausforderung und Chance“ im Dezember 2017 gemeinsam mit Kooperationspartnern und Interessierten die Arbeitsgruppe Forschungsdaten gegründet. Gemeinsam wird an Strategien und Konzepten gearbeitet. Daraus haben sich einige Erkenntnisse ergeben. 


\section{Für eine Zukunft ohne Elefant im Raum}

Praktikable Chancen auf ein nachhaltiges Forschungsdatenmanagement für die Kultur- und Sozialanthropologie und andere mit qualitativen Daten arbeitende sozial- und kulturwissenschaftliche Fächer ergeben sich nur durch disziplinen-, institutionen- und länderübergreifende Strategien. Diese fehlen noch.

Um solche Strategien zu entwickeln, ist ein Kraftakt in personeller und finanzieller Hinsicht, aber auch beim eigenen engen, disziplinen- und institutionengebundenen Denken nötig. Durch die Einbeziehung aller beteiligten Stakeholder auf Augenhöhe (Beforschte, Forschende, Fördergeber, Ministerien, Forschungsstätten, Repositorien, Ethikkommissionen, Nutzerlnnen etc.), durch gezielte Lobbyingarbeit und durch die Schaffung eines geeigneten Rahmens für ein Experimentieren mit und Evaluieren von best practice-Modellen können Richtlinien und Standards für den Umgang mit qualitativen Daten entwickelt werden. Ohne diese Anstrengungen wird der Elefant im Raum bleiben. Ein nachhaltiges Forschungsdatenmanagement für ethnographische und andere qualitative Daten wird dann kaum zu realisieren sein.

Mag. Dr. Igor Eberhard Universität Wien, Institut für Kultur- und Sozialanthropologie

E-Mail: igor.eberhard@univie.ac.at

ao. Univ.-Prof. Dr. Wolfgang Kraus Universität Wien, Institut für Kultur- und Sozialanthropologie E-Mail: wolfgang.kraus@univie.ac.at

\section{Literatur}

AUSSDA (= Austrian Social Science Data Archive) (o.J.): https://aussda.at/ (27.04.2018).

Breidenstein Georg/Hirschauer, Stefan/Kalthoff, Herbert/Nieswand, Boris (2015): Ethnografie: Die Praxis der Feldforschung. 2. Aufl. Konstanz: UVK Verlagsgesellschaft (utb 3979).

Caton, H. (Hg.) (1990): The Samoa Reader: Anthropologists Take Stock. Lanham, MD: University Press of America.

DGSKA ( = Deutsche Gesellschaft für Sozial- und Kulturanthropologie) (2008): „Frankfurter Erklärung“ zur Ethik in der Ethnologie. https://www. 
dgska.de/wp-content/uploads/2016/07/DGV-Ethikerklaerung.pdf (10.07.2018)

EDA (= Ethnographische Datenarchivierung) (o.J.): http://eda.univie.ac.at/ (10.07.2018).

Force11 (2017): https://www.force11.org/group/fairgroup/fairprinciples (10.07.2018).

Imeri, Sabine (2017): Open Data? Zum Umgang mit Forschungsdaten in den ethnologischen Fächern, in Kratzke, Jonas und Heuveline, Vincent (Hg.): E-Science-Tage 2017: Forschungsdaten managen. Heidelberg: heiBOOKS. http://doi.org/10.11588/heibooks.285.377 (10.07.2018).

PRE (= Panel on Research Ethics/Groupe en éthique de la recherche) (2018): TCPS 2 - Chapter 9: Research Involving the First Nations, Inuit and Métis Peoples of Canada. http://www.pre.ethics.gc.ca/eng/policy-politique/initiatives/tcps2-eptc2/chapter9-chapitre9/ (10.07.2018).

Phaidra (o.J.): https://phaidra.univie.ac.at/ (10.07.2018).

RatSWD (= Rat für Sozial- und Wirtschaftsdaten) (2018a): Forschungsdatenmanagement in den Sozial-, Verhaltens- und Wirtschaftswissenschaften: Orientierungshilfen für die Beantragungund Begutachtung datengenerierender und datennutzender Forschungsprojekte. RatSWD Output 3 (5). 2. Aufl. Berlin: Rat für Sozial- und Wirtschaftsdaten. https://doi.org/10.17620/02671.7 (10.07.2018).

RatSWD (= Rat für Sozial- und Wirtschaftsdaten) (2018b): Workshop „Archivierung und Zugang zu qualitativen Daten“. https://www.ratswd.de/ $\mathrm{dl} /$ media/Programm_RatSWD_Workshop_Qualitative_Daten_April_2018_Bremen.pdf(27.04.2018).

Smioski, Andrea (2011): Wegweiser qualitative Datenarchivierung. Infrastruktur, Datenakquise, Dokumentation und Weitergabe, SWS-Rundschau 51 (2): 219-238. http://www.sws-rundschau.at/archiv/SWS_2011_2_ Smioski.pdf(10.07.2018).

Smioski, Andrea (2012): Archivierung und Sekundärnutzung qualitativer Daten. Dissertation, Universität Wien. https://othes.univie.ac.at/26908/ (10.07.2018).

Sterzer, Wjatscheslaw/Imeri, Sabine/Harbeck, Matthias (2018): Zugriff aufethnologische Forschungsdaten: Anforderungen und Lösungen (Konferenzposter). https://nbn-resolving.org/urn:nbn:de:0290-opus4-157193 (10.07.2018).

TIB (= Technische Informationsbibliothek) (2016): Warum braucht man Forschungsdatenmanagement? https://handbuch.tib.eu/w/Warum_braucht_ man_Forschungsdatenmanagement (10.07.2018). 